\title{
Injective mappings and solvable vector fields
}

\author{
JOSÉ R. DOS SANTOS FILHO ${ }^{1}$ and JOAQUIM TAVARES ${ }^{2}$ \\ ${ }^{1}$ Departamento de Matemática, Universidade Federal de São Carlos \\ Via Washington Luis, km 235, 13565-905 São Carlos, SP, Brasil \\ ${ }^{2}$ Departamento de Matemática, Universidade Federal de Pernambuco \\ Av. Prof. Luiz Freire s/n, 50740-540 Recife, PE, Brasil
}

Manuscript received on November 3, 2008; accepted for publication on April 14, 2010

\begin{abstract}
We establish a sufficient condition for injectivity in a class of mappings defined on open connected subsets of $\mathbb{R}^{n}$, for arbitrary $n$. The result relates solvability of the appropriate vector fields with injectivity of the mapping and extends a result proved by the first author for $n \leq 3$. Furthermore, we extend the result to connected paracompact smooth oriented manifolds and show that the convexity condition imposes strong topological restrictions on the manifold.
\end{abstract}

Key words: fields, injectivity, mappings, solvability, vectors.

\section{INTRODUCTION}

Let $\Omega$ be an open connected subset of $\mathbb{R}^{n}$, and consider $\Phi(\Omega)=\left\{\mathrm{F} \in C^{\infty}\left(\Omega, \mathbb{R}^{n}\right)\right.$ : $\left.\operatorname{det}(\mathrm{DF})(x) \neq 0 \forall x\right\}$. We denote an element of $\Phi(\Omega)$ by $\mathrm{F}=\left(\mathrm{f}_{1}, \ldots, \mathrm{f}_{n}\right)$. A very basic problem in Mathematical Analysis is to determine additional conditions under which $\mathrm{F}$ is injective. The most famous related problem is the so called Jacobian Conjecture, which states that for a polynomial mapping on $\mathbb{C}^{n}$ no additional condition is necessary, see (Bass et al. 1982). For applications and related bibliography see (Santos Filho 2004). As in Santos Filho, for each $i \in\{1,2, \ldots, n\}$, we consider $\mathcal{V}_{\mathrm{F}, i}$ the real vector field defined by

$$
\left.\mathcal{V}_{\mathrm{F}, i}(\phi)(x)=\operatorname{det}\left(\mathrm{DF}_{i, \phi}\right)(x)\right) \text {, for all } \phi \in C^{\infty} \text {. }
$$

Here the mapping $\mathrm{F}_{i, \phi}$ is given by: Its $\mathrm{j}$-component is equal to $\mathrm{f}_{j}$ if $j \neq i$ and its $\mathrm{i}$-component is equal to $\phi$. It follows that the non-empty connected components of $\left\{x \in \Omega ; \mathrm{f}_{j}(x)=c_{j}, j \neq i\right\}$, where $c_{1}, \ldots, c_{i-1}, c_{i+1}, \ldots, c_{n} \in \mathbb{R}$, defines a smooth one-dimensional foliation of $\Omega$. This foliation is precisely that one of the characteristic curves of $\mathcal{V}_{\mathrm{F}, i}$, because each $\mathrm{f}_{j}$ is a first integral of $\mathcal{V}_{\mathrm{F}, i}$, if $j \in\{1, \ldots, n\}$ with $j \neq i$. In order to characterize solvability of linear partial differential operators

Correspondence to: José R. dos Santos Filho

E-mail: santos@dm.ufscar.br 
JOSÉ R. DOS SANTOS FILHO and JOAQUIM TAVARES

on a manifold, in (Malgrange 1956), B. Malgrange introduced a notion of convexity, namely the condition (a)(2) of Theorem 2.1 below. As in (Santos Filho 2004), a similar notion is in order.

Definition 1.1. Let $\mathrm{F} \in \Phi(\Omega)$. We say that $\Omega$ is F-convex if there are an open set of $\Omega_{1}$ of $\mathbb{R}^{n}$, $\mathrm{G}_{1} \in \Phi\left(\Omega_{1}\right)$ with $\mathrm{G}_{1}\left(\Omega_{1}\right)=\Omega$, and $\mathrm{G}_{2} \in \Phi(\mathrm{F}(\Omega))$ where, for $j=1,2$, each $\mathrm{G}_{j}$ is a diffeomorphism over its image such that there are $n-1$ different indices $i_{1}, \ldots, i_{n-1} \in\{1, \ldots, n\}$ where $\Omega_{1}$ is $\mathcal{V}_{F_{1}, i_{j}}$-convex for $j \in\{1, \ldots, n-1\}$ and $\mathrm{F}_{1}=\mathrm{G}_{2} \circ \mathrm{F} \circ \mathrm{G}_{1}$.

For $n=2,3$ it was proved in Theorems 0.1 and Theorem 0.2 of (Santos Filho 2004), that: If $\Omega$ is F-convex then $\mathrm{F}$ is injective. Also $\Omega$ is simply connected when $n=2$.

Here we give a full generalization of these results. We mean not only for arbitrary dimensions, but also for the smooth category of oriented manifolds too. Our main goal is to generalize, for higher dimensions and arbitrary manifolds, those results. First we address the euclidean case as a introduction in Theorem 1.1 and then we furnish the needed tools for the general case in Theorem 2.2 below.

THEOREM 1.1. Let $\Omega$ be an open connected subset of $\mathbb{R}^{n}$ and $\mathrm{F} \in \Phi(\Omega)$. If $\Omega$ is $\mathrm{F}$-convex then $\mathrm{F}$ is injective.

EXAMPLE. Consider the smooth mapping of the plane given by $\mathrm{F}(x, y)=\left(x, y \exp x^{-2}\right)$ for $x \neq 0$ and $\mathrm{F}(0, y)=(0,0)$ if $x=0$. Then $\mathcal{V}_{\mathrm{F}, 2}=\partial / \partial y$, so $\mathbb{R}^{2}$ is $\mathrm{F}$-convex. Moreover $\operatorname{det}(\mathrm{DF}(x))=\exp \left(x^{-2}\right)>0$ if $x \neq 0$, so is positive except at a closed set of null Lebesgue measure, in the other hand $\mathrm{F}$ is not injective. So the condition of $F \in \Phi\left(\mathbb{R}^{2}\right)$ in Theorem 1.1 can not be in this fashion.

This paper is organized in the following way: First we prove Theorem 1.1, then extend it for connected paracompact smooth oriented n-dimensional manifold $M$ and finally show that $M$ must be contractible if $M$ is F-convex for some $\mathrm{F} \in \Phi\left(M, \mathbb{R}^{n}\right)$. We conclude by making some remarks regarding the results.

\section{PROOFS OF THE RESULTS AND REMARKS}

Before we prove our theorems we recall part of Theorem 6.4.2 of Duistermaat and Hörmander, in (Duistermaat and Hörmander 1972), this result characterizes global solvability of vector fields considered as partial differential operators:

THEOREM 2.1. Let $L$ be a smooth real vector field of a $C^{\infty}$ manifold $M$, the following conditions (a) and (b) below are equivalent:

(a) (1) No complete integral curve of $L$ is contained in compact subset of $M$.

(2) For every compact subset $K$ of $M$ there exists a compact subset $K^{\prime}$ of $M$ such that every compact interval of an integral curve with end points in $K$ is contained in $K^{\prime}$.

(b) There exists a manifold $M_{0}$ and an open neighborhood $M_{1}$ of $M_{0} \times\{0\}$ in $M_{0} \times \mathbb{R}$ which is convex in the $\mathbb{R}$ direction, and a diffeomorphism from $M \rightarrow M_{1}$ which carries $L$ into the operator $\partial / \partial t$ if points of $M_{0} \times \mathbb{R}$ are denoted by $\left(y_{0}, t\right)$.

In the first subsection we prove Theorem 1.1, in the second subsection we state and prove an extension of it. Then a topological consequence, as corollary, is deduced and remarks are made. 


\section{Proof OF Theorem 1.1}

Without loss of generality we can assume that $\Omega_{1}=\Omega$ and $i_{j}=j$ for $j=1, \ldots, n-1$. Our proof follows by a finite induction argument once we prove injectivity for appropriate restrictions of $F$. Let $j$ an integer, $1 \leq j \leq n-1$, and $\left(c_{1}, \ldots, c_{n}\right) \in \operatorname{Image}(\mathrm{F})$, consider $M_{j}$ be a connected component of $\cap_{i=1}^{j} \mathbf{f}_{i}^{-1}\left(\left\{c_{i}\right\}\right)$. From the hypotheses, it follows that for each $j M_{j}$ is a $C^{\infty}$ submanifold of dimension $n-j$ of $\Omega$, which is invariant by the flow of of $\mathcal{V}_{\mathrm{F}, i}$, for $j+1 \leq i \leq n$.

Let $j \in\{1,2, \ldots, n\}$, since $\mathcal{V}_{\mathrm{F}, j}\left(f_{i}\right)=\operatorname{det}(\mathrm{DF}(x)) \delta_{i j}$ where $\delta_{i j}$ is the Kronecker symbol, we have that both $\omega$-limit and $\alpha$-limit of characteristic curves of $\mathcal{V}_{\mathrm{F}, j}$ are empty. Hence condition (a)(1) of Theorem 2.1 is true for $L=\mathcal{V}_{\mathrm{F}, j}$, if $j \in\{1, \ldots, n-1\}$. Therefore, in our case, condition (a) of Theorem 2.1 is equivalent (a)(2), which it is exactly the meaning of $\Omega$ be $\mathcal{V}_{F, i_{j}}$-convex. So from Theorem 1.1 we have that (b) holds, that is:

For each $1 \leq j \leq n-1$, the following holds

$(*)_{j}$ There exist a manifold $M_{0, j}$, an open neighborhood $M_{1, j}$ of $M_{0, i} \times\{0\}$ in $M_{0, j} \times \mathbb{R}$ which is convex in the $\mathbb{R}$ direction, and a diffeomorphism $\Omega \rightarrow M_{1, j}$ which carries each $\mathcal{V}_{\mathrm{F}, j}$ into the operator $\partial / \partial t$ if points in $M_{0, j} \times \mathbb{R}$ are denoted by $\left(y_{0, j}, t\right)$.

The next step of the proof is to show that we can take $M_{0, j}$ so that its image on $\Omega$, by the diffeomorphism of $(*)_{j}$, is equal to a connected component of a level set of $\mathrm{f}_{j}$. In order to prove this, consider $X_{i}=\left(\operatorname{det}\left(\mathrm{F}^{\prime}(x)\right)^{-1} \mathcal{V}_{\mathrm{F}, i}\right.$, for $i=1, \ldots, n$, so $\left[X_{i}, X_{j}\right]\left(\mathrm{f}_{k}\right)=0$ for all $i, j$ and $k$, therefore by the Inverse Function Theorem we have that $\left[X_{i}, X_{j}\right]=0$ for all $i$ and $j$. Moreover, the orbits of $X_{i}$, for $1 \leq i \leq n$ are equal to the corresponding $\mathcal{V}_{\mathrm{F}, i}$.

So for $1 \leq j \leq n-1(*)_{j}$ holds for $X_{j}$ as well. From Frobenius Theorem, since the $X_{j}$ 's commute, the image on $\Omega$ of $M_{0, j}$ by the diffeomorphism must be orthogonal to $X_{j}$. Therefore it is a connected component of a pre-image of $\mathrm{f}_{j}$, and the same holds for $\mathcal{V}_{\mathrm{F}, j}$.

Assume that there are two points $p_{1}$ and $p_{2}$ of $M$ such that $\mathrm{F}\left(p_{1}\right)=\mathrm{F}\left(p_{2}\right)$, in particular we have that $\mathrm{f}_{1}\left(p_{1}\right)=\mathrm{f}_{1}\left(p_{2}\right)$. Clearly if the characteristic curves of $\mathcal{V}_{\mathrm{F}, 1}$ passing through each $p_{i}$ are the same then we have that $p_{1}=p_{2}$, because $\mathcal{V}_{\mathrm{F}, 1}\left(\mathrm{f}_{1}\right) \neq 0$. So we assume that the above curves are different connected components of $\left\{x ; \mathrm{f}_{j}(x)=\mathrm{f}_{j}\left(p_{1}\right)\right.$, for $\left.j \neq 1\right\}$.

Identifying $M_{0,1}$ with its image by the diffeomorphism given in $(*)_{j}$, by the above it must be equal to a connected component of $\mathrm{f}_{1}^{-1}\left(c_{1}\right)\left(=M_{0,1}\right)$, for some real $c_{1}$. Furthermore, since $\left\{\mathcal{V}_{\mathrm{F}, i} ; 2 \leq i \leq n\right\}$ are tangent to $\mathrm{f}_{1}^{-1}(c)$, we reduce to prove the theorem for $\left.\mathrm{F}\right|_{M_{0,1}} \in \Phi\left(M_{0,1}, \mathbb{R}^{n-1}\right)$.

We apply the argument above until we get the restriction of $\mathrm{F}$ to a component connected of $\cap_{1 \leq j \leq n-1} \mathrm{f}_{j}^{-1}\left(c_{j}\right)$, but on this curve the restriction of $\mathbf{F}$ is equal to $\mathrm{f}_{n}$. So $\mathbf{F}$ must be injective there by using that $\mathcal{V}_{\mathrm{F}, n} \mathrm{f}_{n} \neq 0$. Concluding the proof of Theorem 0.1 .

\section{EXTENSION OF THEOREM 1.1}

Now we consider an extension of Theorem 1.1 for a connected paracompact smooth oriented $n$-dimensional manifold $(M, \omega)$, where $\omega$ is a globally defined non-vanishing smooth $n$-form. We refer to (Warner 1983 ) for details. 
Suppose that $\mathrm{F}=\left(\mathrm{f}_{1}, \ldots, \mathrm{f}_{n}\right): M \rightarrow \mathbb{R}^{n}$ is a smooth mapping with injective derivative at any point of $M$. As before such a mapping is said to belong to $\Phi(M)=\Phi\left(M, \mathbb{R}^{n}\right)$. From the hypothesis on $M$ we have that there is $\left(U_{m}, \varphi_{m}\right)_{m \in \mathbb{Z}_{+}}$a coordinate system for $M$ so that $U_{m}$ is pre-compact for each $m$. Take $\left(\psi_{m}\right)_{m \in \mathbb{Z}_{+}}$to be a partition of unity associated to $\left(U_{m}\right)$. Furthermore, we can assume that the restriction of $\mathrm{F}$ on $U_{m}$ is a diffeomorphism over its image.

Let $1 \leq j \leq n$ consider the vector field $\mathcal{V}_{j}$ defined as follows, let $g \in C^{\infty}(M)$, take:

$$
\mathcal{V}_{j}(g)=\operatorname{coeff}\left[(-1)^{n-j} d \mathrm{f}_{1} \wedge \ldots \wedge\left(d \mathrm{f}_{j}\right)^{v} \wedge \ldots \wedge d \mathrm{f}_{n} \wedge d(g) \text { in terms of } \omega\right]
$$

It is a routine computation to show that $\mathcal{V}_{j}$ is a derivation, then it defines a vector field on $M$.

Now we will see that these vector fields generalize for $M$ the vector fields considered before on the the euclidean context. In fact, let $g \in C^{\infty}(M)$ and write $g=\Sigma_{m} \psi_{n} g$, therefore the support of $\psi_{m} g$ is contained on $U_{m}$. Since the restriction of $\mathrm{F}$ to $U_{m}$ is a diffeomorphism we can find a $g_{m} \in C_{c}^{\infty}\left(\mathrm{F}\left(U_{m}\right)\right)$ such that $\psi_{m} g=g_{m} \circ$ F. So $g=\Sigma_{m} g_{m} \circ$ F. Then

$$
\left[(-1)^{n-j} d \mathrm{f}_{1} \wedge \cdots \wedge\left(d \mathrm{f}_{j}\right)^{v} \wedge \cdots \wedge d \mathrm{f}_{n} \wedge d(g)\right]=\Sigma_{m} \partial_{n} g_{m} d \mathrm{f}_{1} \wedge \cdots \wedge d \mathrm{f}_{n} .
$$

Defining $\operatorname{Det}(D \mathrm{~F}(x))$ to be the coefficient of $d \mathrm{f}_{1} \wedge \cdots \wedge d \mathrm{f}_{n}$ in terms of $\omega$, we have that

$$
\mathcal{V}_{j}(g)=\Sigma_{m} \partial_{n} g_{m} \operatorname{Det}(D F(x)) .
$$

As before we have that $\mathrm{f}_{j}$, for $j \neq i$, is a first integral of $\mathcal{V}_{i}$. Also the vector fields are in involution. We consider an extension of Definition 1.1:

Definition 2.1. Let $\mathrm{F} \in \Phi(M)$. We say that $M$ is F-convex if there are an smooth manifold $M_{1}$, $\mathrm{G}_{1} \in \Phi\left(M_{1}, M\right)$ with $\mathrm{G}_{1}\left(M_{1}\right)=M$ and $\mathrm{G}_{2} \in \Phi(\mathrm{F}(\Omega))$ where, for $j=1,2$, each $\mathrm{G}_{j}$ is a diffeomorphism over its image such that there are $n-1$ different indices $i_{1}, \ldots, i_{n-1} \in\{1, \ldots, n\}$ where $M_{1}$ is $\mathcal{V}_{F_{1}, i_{j}}$-convex for $j \in\{1, \ldots, n-1\}$ and $\mathrm{F}_{1}=\mathrm{G}_{2} \circ \mathrm{F} \circ \mathrm{G}_{1}$.

From inspection on the proof of Theorem 1.1 we have:

THEOREM 2.2. Let $M$ be a connected paracompact smooth oriented manifold and $\mathrm{F} \in \Phi(M)$. If $M$ is $\mathrm{F}$-convex then $\mathrm{F}$ is injective.

Theorem 2.2 could be used to decide whether a local parametrization of a manifold, defined globally, is a global parametrization. The result below extends for arbitrary dimension Theorem 0.3 of (Santos Filho 2004):

COROLLARY 2.1. Let $M$ be be a connected paracompact smooth oriented manifold and $\mathrm{F} \in \Phi(M)$ so that $\Omega$ is F-convex. Then $M$ is contractible.

We observe from the proof of Theorem 1.1 that $M$ is a diffeomorphic image of $\gamma \times \mathbb{R}^{n-1}$ where $\gamma$ is a smooth curve which is diffeomorphic to an interval. Then the conclusion of the corollary follows. 


\section{REMARKS.}

1) The proof that each pair of $\mathcal{V}_{\mathrm{F}, j}$ 's is in involution can also be proved without normalizing them. That is without considering the $X_{j}^{\prime} s$. In fact, let $\mathcal{V}_{\mathrm{F}, j_{1}}$ and $\mathcal{V}_{\mathrm{F}, j_{2}}$, with $j_{1} \neq j_{2}$, then $\mathcal{V}_{\mathrm{F}, j_{i}}\left(f_{j}\right)=0$ if $j \neq j_{i}$ for $i \in\{1,2\}$. Therefore $\left[\mathcal{V}_{\mathrm{F}, j_{1}}, \mathcal{V}_{\mathrm{F}, j_{2}}\right]$ at any point is linear combination of $\mathcal{V}_{\mathrm{F}, j_{1}}$ and $\mathcal{V}_{\mathrm{F}, j_{2}}$, proving the assertion.

2) From Theorem 2.1, we can not have a vector field globally solvable on a compact manifold without boundary. Otherwise the manifold would be a diffeomorphic image of a cylinder $M_{0} \times \mathbb{R}$, where $M_{0}$ is a $n-1$ dimensional manifold, therefore imposing a strong restriction on the topology of $M$.

\section{ACKNOWLEDGMENTS}

This work was partially supported by the Grant no. 2007/08231-0 from Fundação de Amparo à Pesquisa do Estado de São Paulo (FAPESP) to José R. dos Santos Filho.

\section{RESUMO}

Nós estabelecemos uma condição suficiente para injetividade numa classe de aplicações definidas em subconjuntos abertos conexos de $\mathbb{R}^{n}$, para $n$ arbitrário. O resultado relaciona resolubilidade de campos de vetores apropriados com injetividade da aplicação e estende o resultado demonstrado pelo primeiro autor quando $n \leq 3$. Além disso, nós estendemos o resultado para variedades suaves orientadas e para-compactas e mostramos que a condição de convexidade impõe fortes restrições topológicas na variedade.

Palavras-chave: campos, injetividade, aplicações, resolubilidade, vetores.

\section{REFERENCES}

Bass H, CONNEll EH AND Wright D. 1982. The Jacobian conjecture: Reduction of degree and formal expansion of the inverse. Bull Am Math Soc, New Ser 7: 287-330.

DuistermaAt JJ AND Hörmander L. 1972. Fourier Integral Operators II. Acta Math 128: 183-269.

Malgrange B. 1956. Existence et approximation des solutions des équations aux dérivées partielles et des équations de convolution. Ann Inst Fourier (Grenoble) 6: 271-355.

SANTOS FiLHo JR DOS. 2004. Injective mappings and solvable vector fields of Euclidean Spaces. Topology Appl 136: $261-274$.

WARNER F. 1983. Foundations of differentiable manifolds and Lie groups. Springer-Verlag. 